\title{
Existence of Solutions of a Non-Linear Eigenvalue Problem with a Variable Weight
}

\author{
Rejeb Hadiji, François Vigneron
}

June 19, 2018

Université Paris-Est, Laboratoire d'Analyse et de Mathématiques Appliquées, UMR 8050 du CNRS 61, avenue du Général de Gaulle, F-94010 Créteil - France.

\begin{abstract}
We study the non-linear minimization problem on $H_{0}^{1}(\Omega) \subset L^{q}$ with $q=\frac{2 n}{n-2}, \alpha>0$ and $n \geq 4$ :

$$
\inf _{\substack{u \in H_{0}^{1}(\Omega) \\\|u\|_{L^{q}=1}}} \int_{\Omega} a(x, u)|\nabla u|^{2}-\lambda \int_{\Omega}|u|^{2} .
$$

where $a(x, s)$ presents a global minimum $\alpha$ at $\left(x_{0}, 0\right)$ with $x_{0} \in \Omega$. In order to describe the concentration of $u(x)$ around $x_{0}$, one needs to calibrate the behaviour of $a(x, s)$ with respect to $s$. The model case is

$$
\inf _{\substack{u \in H_{0}^{1}(\Omega) \\\|u\|_{L} q=1}} \int_{\Omega}\left(\alpha+|x|^{\beta}|u|^{k}\right)|\nabla u|^{2}-\lambda \int_{\Omega}|u|^{2} .
$$

In a previous paper dedicated to the same problem with $\lambda=0$, we showed that minimizers exist only in the range $\beta<k n / q$, which corresponds to a dominant non-linear term. On the contrary, the linear influence for $\beta \geq k n / q$ prevented their existence. The goal of this present paper is to show that for $0<\lambda \leq \alpha \lambda_{1}(\Omega), 0 \leq k \leq q-2$ and $\beta>k n / q+2$, minimizers do exist.
\end{abstract}

Keywords : Critical Sobolev exponent, Minimization problem, Non-linear effects.

AMS classification : 35A01, 35A15, 35J57, 35J62.

\section{Introduction, notations and statement of the result}

\subsection{The classical non-linear problem}

The domain $\Omega$ is a smooth, bounded subset of $\mathbb{R}^{n}$ with $n \geq 4$. Let us recall the traditional minimization problem of $[7]$ :

$$
S=\inf _{\substack{u \in H_{0}^{1}(\Omega) \\\|u\|_{L^{q}}=1}} \int_{\Omega}|\nabla u|^{2}
$$

where $q=\frac{2 n}{n-2}$ is the critical exponent for the Sobolev embedding $H_{0}^{1}(\Omega) \subset L^{q}(\Omega)$. For a smooth positive cut-off function $\zeta$ compactly supported and equal to 1 near the origin, a minimizing sequence for $S$ is given by $\omega_{\varepsilon} /\left\|\omega_{\varepsilon}\right\|_{L^{q}}$ as $\varepsilon \rightarrow 0$ with

$$
\omega_{\varepsilon}(x)=\frac{\varepsilon^{\frac{n-2}{4}} \zeta(x)}{\left(\varepsilon+|x|^{2}\right)^{\frac{n-2}{2}}} .
$$


According to [7], $S$ is never achieved but one has $S=K_{1} / K_{2}$ where the constants $K_{1}$ and $K_{2}$ are the limit of the $H_{0}^{1}$ and $L^{q}$ norm of $\omega_{\varepsilon}$ :

$$
K_{1}=\lim _{\varepsilon \rightarrow 0}\left\|\nabla \omega_{\varepsilon}\right\|_{L^{2}}^{2} \quad \text { and } \quad K_{2}=\lim _{\varepsilon \rightarrow 0}\left\|\omega_{\varepsilon}\right\|_{L^{q}}^{2} .
$$

For example, the computation for the gradient goes as follows:

$$
\nabla \omega_{\varepsilon}(x)=-(n-2) \frac{\varepsilon^{\frac{n-2}{4}} x \zeta(x)}{\left(\varepsilon+|x|^{2}\right)^{\frac{n}{2}}}+\frac{\varepsilon^{\frac{n-2}{4}} \nabla \zeta(x)}{\left(\varepsilon+|x|^{2}\right)^{\frac{n-2}{2}}} .
$$

As $\nabla \zeta=0$ in a neighborhood of the origin, one gets

$$
\left|\nabla \omega_{\varepsilon}(x)\right|^{2} \underset{|x| \rightarrow 0}{\sim}(n-2)^{2} \varepsilon^{\frac{n-2}{2}} \frac{|x|^{2} \zeta^{2}(x)}{\left(\varepsilon+|x|^{2}\right)^{n}},
$$

which integrates to a constant independent of $\varepsilon$. In a similar way, one can deduce precise asymptotics for the various norms, that we will reuse later (see again [7]):

$$
\begin{gathered}
\int_{\Omega}\left|\nabla \omega_{\varepsilon}(x)\right|^{2} d x=K_{1}+O\left(\varepsilon^{\frac{n-2}{2}}\right), \quad\left(\int_{\Omega} \omega_{\varepsilon}^{q}(x) d x\right)^{2 / q}=K_{2}+O\left(\varepsilon^{\frac{n-2}{2}}\right), \\
\int_{\Omega} \omega_{\varepsilon}^{2}(x) d x= \begin{cases}K_{3} \varepsilon|\log \varepsilon|+O(\varepsilon) & \text { if } n=4 \\
K_{3} \varepsilon+O\left(\varepsilon^{\frac{n-2}{2}}\right) & \text { if } n \geq 5 .\end{cases}
\end{gathered}
$$

\subsection{The general non-linear problem}

We are interested in the following non-linear minimization problem:

$$
S_{\lambda}(a)=\inf _{\substack{u \in H_{0}^{1}(\Omega) \\\|u\|_{L^{q}=1}}}\left\{\int_{\Omega} a(x, u)|\nabla u|^{2}-\lambda \int_{\Omega}|u|^{2}\right\} .
$$

where $a(x, s)=a(x,-s)$ is a smooth function, for example continuous on $\Omega \times \mathbb{R}$, with a continuous derivative with respect to $s$ on $\Omega \times \mathbb{R}_{+}$. We assume that $a(x, s)$ presents a global minimum $\alpha$ at $\left(x_{0}, 0\right)$ with $x_{0} \in \Omega$. One expects that minimizing sequences $u_{j}(t, x)$ will concentrate around $x_{0}$. In order to describe the concentration of $u(x)$ around $x_{0}$, one needs to calibrate the behaviour of $a(x, s)$ with respect to $s$.

The model case we are focusing on is

$$
a(x, s)=b_{1}(x)+b_{2}(x)|s|^{k}
$$

where $b_{1}(x) \geq \alpha=b_{1}\left(x_{0}\right)$ and $b_{2}(x) \geq 0$ with $b_{2}\left(x_{0}\right)=0$. Even in this simplified model, changes to the value of $k$ or of the behaviours of $b_{1}$ or $b_{2}$ around $x_{0}$ can lead to radically different phenomena for the minimization problem (7). The proper assumptions are the following:

1. The exponent $k$ satisfies

$$
0 \leq k<q
$$

This range will be slightly reduced in the statement of Theorem 3 and we will explain why in the next subsection.

2. $b_{1}$ has a global minimum $\alpha=b_{1}\left(x_{0}\right)$ at some point $x_{0} \in \Omega$, of order $\gamma>2$, i.e.:

$$
\left\{\begin{array}{l}
b_{1}(x)=\alpha+O\left(\left|x-x_{0}\right|^{\gamma}\right), \quad \gamma>2 \\
b_{1}(x) \geq \alpha \quad \text { if } \quad x \neq x_{0}
\end{array}\right.
$$


3. $b_{2}$ is positive and has a unique zero in $\Omega$ at the same point $x_{0}$, of order $\beta \geq 0$

$$
\left\{\begin{array}{l}
b_{2}(x)=\left|x-x_{0}\right|^{\beta}+o\left(\left|x-x_{0}\right|^{\beta}\right) \\
b_{2}(x)>0 \text { if } \quad x \neq x_{0} .
\end{array}\right.
$$

Further restrictions on the value of $\beta$ will be explained in the next subsection.

For the sake of clarity, we are going to focus most of this paper on the reduced model where $b_{1}$ is a constant and $b_{2}$ is a power law. Without restrictions, one can also assume that $x_{0}$ is the origin. One is thus lead to the following reduced minimization problem:

$$
S_{\lambda}(\beta, k)=\inf _{\substack{u \in H_{0}^{1}(\Omega) \\\|u\|_{L^{q}}=1}} E_{\lambda}(u)
$$

where

$$
E_{\lambda}(u)=\int_{\Omega}\left(\alpha+|x|^{\beta}|u|^{k}\right)|\nabla u|^{2}-\lambda \int_{\Omega}|u|^{2} .
$$

The case $\lambda=0$ has been extensively studied in our previous paper, [21]. The case $k=0$ has already been dealt with in [23].

The general model case (8) will be detailed in the last section, $\S 4$, of this paper. In its full generality, the qualitative properties of minimizing sequences of (7) are too varied to be described easily.

\subsubsection{Natural scaling(s) of the problem}

For the sake of clarity, let us discuss the various natural possible scalings on the reduced problem (12). Depending on the ratio $\beta / k$, different situations occur in the blow-up scale around the point where the weight is singular. More precisely, let us define $v_{\varepsilon}$ by $v(x)=\varepsilon^{-n / q} v_{\varepsilon}(x / \varepsilon)$ and $\Omega_{\varepsilon}=\varepsilon^{-1} \Omega$.

- If $\beta<\frac{k n}{q}$ the leading term of the blow-up around $x=0$ is the non-linear one:

$$
E_{0}(v) \underset{\varepsilon \rightarrow 0}{\sim} \varepsilon^{-\left(\frac{k n}{q}-\beta\right)} \int_{\Omega_{\varepsilon}}|y|^{\beta}\left|v_{\varepsilon}(y)\right|^{k}\left|\nabla v_{\varepsilon}(y)\right|^{2} d y .
$$

We showed in [21] that $E_{0}$ admits minimizers on $\mathbb{S}=\left\{u \in H_{0}^{1}(\Omega) \mid\|u\|_{L^{q}}=1\right\}$ in this case.

- If $\beta=\frac{k n}{q}$ the first linear and non-linear terms have the same weight and the blow-up rescaling leaves the value of $E_{0}(v)$ unchanged. The corresponding infimum $S_{0}(\beta, k)$ does not depend on $\Omega$ but $E_{0}$ admits no smooth minimizer on $\mathbb{S}$.

- If $\beta>\frac{k n}{q}$, the blow-up around $x=0$ gives

$$
E_{\lambda}(v)=\alpha \int_{\Omega_{\varepsilon}}\left|\nabla v_{\varepsilon}(y)\right|^{2} d y+\varepsilon^{\left(\beta-\frac{k n}{q}\right)} \int_{\Omega_{\varepsilon}}|y|^{\beta}\left|v_{\varepsilon}(y)\right|^{k}\left|\nabla v_{\varepsilon}(y)\right|^{2} d y-\varepsilon^{2} \lambda \int_{\Omega_{\varepsilon}} v_{\varepsilon}^{2}(y) d y .
$$

When $\beta>\frac{k n}{q}$ and $\lambda=0$, we have shown in [21] that the linear behavior is dominant and that $E_{0}$ admits no minimizer on $\mathbb{S}$. One can even find a common minimizing sequences for both the linear and the non-linear problem. A cheap way to justify this is as follows. The problem tends to concentrate $u$ as a radial decreasing function around the origin. Thus, when $\beta>k n / q$, one can expect $|u(x)|^{q} \ll 1 /|x|^{\beta q / k}$ because the right-hand side would not be locally integrable while the left-hand side is required to. In turn, this inequality reads $|x|^{\beta}|u(x)|^{k} \ll 1$, which eliminates the non-linear contribution in the minimizing problem $E_{0}$. 
When $\lambda \neq 0$, the situation is quite different as both linear terms compete. We will show in this paper that if $\beta>\frac{k n}{q}+2$, the problem of minimizing $E_{\lambda}(u)$ admits solutions. This result has the same flavor as [7] but even if the non-linear term is not expected to be dominant, one has to deal with it rigorously.

The gap between our existence result in [21] (namely $\beta<k n / q$ ) and Theorem 3 below (i.e. $\beta>k n / q+2)$ cannot be bridged easily. For $\beta<\frac{k n}{q}$, we have shown in [21] that $E_{0}(u)$ admits minimizers. It is natural to expect that $E_{\lambda}(u)$ would too, as a perturbation problem. For $\beta>\frac{k n}{q}+2$, $E_{0}(u)$ does not admit minimizers, but according to Theorem 3 below, $E_{\lambda}(u)$ does for $\lambda>0$ small enough. What happens in the case of $\frac{k n}{q} \leq \beta \leq \frac{k n}{q}+2$ is not known. Let us however point out that, in the case of $\beta=2$ and $0 \leq k \leq \frac{4}{n-2}$ which is a particular instance of $\frac{k n}{q} \leq \beta \leq \frac{k n}{q}+2$, the Pohožaev identity (see e.g. [28] or [21, eq. 18])

$$
\frac{1}{2}\left(\beta-\frac{k n}{q}\right) \int_{\Omega}|x|^{\beta}|u|^{k}|\nabla u|^{2}+\frac{1}{2} \int_{\partial \Omega}\left|\frac{\partial u}{\partial \nu}\right|^{2}(x \cdot \mathbf{n})=\lambda \int_{\Omega} u^{2}
$$

This ineqality can be restrictive for $\lambda$. For instance, if $\Omega$ is star-shaped with respect to the origin, then $x \cdot \mathbf{n} \geq 0$. Combined with a Hardy type inequality (see for example [9] or [23]), it then gives us:

$$
\frac{n^{2}}{8}\left(2-\frac{k n}{q}\right)\left(\frac{k}{2}+1\right)^{-2} \int_{\Omega}|u|^{k+2} \leq \frac{1}{2}\left(2-\frac{k n}{q}\right)\left(\frac{k}{2}+1\right)^{-2} \int_{\Omega}\left|x \cdot \nabla\left(u^{\frac{k}{2}+1}\right)\right|^{2} \leq \lambda \int_{\Omega} u^{2} .
$$

If, for example, $k=0$, the solution $u$ does not exist if $\lambda<\frac{n^{2}}{4}$.

Actually, the blow-up picture (14) is slightly more complicated than just saying that the non-linear term disappears because it is associated to a high power of $\varepsilon \ldots$ As we know, the Sobolev embedding grants $H_{0}^{1} \subset L^{q} \cap L^{2}$. But because of the identity

$$
\int_{\Omega}|x|^{\beta}|u|^{k}|\nabla u|^{2}=\left(\frac{k}{2}+1\right)^{-2} \int_{\Omega}|x|^{\beta}\left(\nabla\left(|u|^{\frac{k}{2}+1}\right)\right)^{2},
$$

the uniform boundedness of the non-linear term (as one can expect along a minimizing sequence of (12)) implies an additional restriction on $u$, namely roughly $u^{\frac{k}{2}+1} \in L^{q}$ i.e. $u \in L^{\bar{q}}$ with $\bar{q}=$ $q /(k / 2+1)$. On a bounded domain $\Omega$, this information does not seem relevant because it was already granted by the Hölder inequality $u \in L^{q}(\Omega) \subset L^{\bar{q}}(\Omega)$. But in the blow-up process, the domain $\Omega_{\varepsilon}=\varepsilon^{-1} \Omega$ is ultimately rescaled to $\mathbb{R}^{n}$ and the non-linear restriction then takes on its full significance. For example, it could happen in (14) that $v_{\varepsilon}$ blows up to a function that does not belong to $L^{\bar{q}}\left(\mathbb{R}^{n}\right)$. In that case, the non-linear term

$$
\varepsilon^{\left(\beta-\frac{k n}{q}\right)} \int_{\Omega_{\varepsilon}}|y|^{\beta}\left|v_{\varepsilon}(y)\right|^{k}\left|\nabla v_{\varepsilon}(y)\right|^{2} d y=C_{k} \varepsilon^{\left(\beta-\frac{k n}{q}\right)} \int_{\Omega_{\varepsilon}}|y|^{\beta}\left|\nabla\left(v_{\varepsilon}(y)^{\frac{k}{2}+1}\right)\right|^{2} d y
$$

might not be of a lower order anymore and the nature of the problem would then change completely. To avoid this potentially disastrous effect, one needs to ensure that $L^{q}\left(\mathbb{R}^{n}\right) \cap L^{2}\left(\mathbb{R}^{n}\right)$ is embedded in $L^{\bar{q}}\left(\mathbb{R}^{n}\right)$. This restriction reads simply $\bar{q} \geq 2$ and boils down to

$$
k \leq q-2 .
$$

This will be part of the assumptions in Theorem 3 . When on the contrary $k>q-2$, it is not clear that the non-linear term is a lower-order term in the blow-up scaling (14). 


\subsubsection{Admissible values for $\lambda$}

In the minimization problem (12)-(13), the critical value for $\lambda$ is the first Dirichlet eigenvalue:

$$
\lambda_{1}(\Omega)=\inf _{\substack{u \in H_{0}^{1} \\\|u\|_{L^{2}}=1}} \int_{\Omega}|\nabla u|^{2} .
$$

Let us recall that $\alpha>0$ is the minimum value of the weight in our energy functional (13).

Proposition 1 For $0 \leq \lambda \leq \alpha \lambda_{1}(\Omega)$ and $k>0$, one has

$$
S_{\lambda}(\beta, k) \geq 0 .
$$

When $\lambda<\alpha \lambda_{1}(\Omega)$, one even has $S_{\lambda}(\beta, k)>0$.

Proof. Let us define:

$$
\lambda_{1}^{\beta, k}(\Omega)=\inf _{\substack{u \in H_{0}^{1}(\Omega) \\ u \neq 0}} \frac{\int_{\Omega}\left(\alpha+|x|^{\beta}|u|^{k}\right)|\nabla u|^{2}}{\int_{\Omega} u^{2}} .
$$

First, let us check that $\lambda_{1}^{\beta, k}(\Omega)=\alpha \lambda_{1}(\Omega)$. Indeed, let $\varphi \in H_{0}^{1}(\Omega)$ such that $-\Delta \varphi=\lambda_{1}(\Omega) \varphi$ with $\varphi \neq 0$. Then, if one uses $\frac{1}{N} \varphi(x)$ as a test function with $N \in \mathbb{N}$, one gets, for $k>0$ :

$$
0<\alpha \lambda_{1}(\Omega) \leq \lambda_{1}^{\beta, k}(\Omega) \leq \alpha \lambda_{1}(\Omega)+\frac{1}{N^{k}} \frac{\int_{\Omega}|x|^{\beta}|\varphi|^{k}|\nabla \varphi|^{2}}{\int_{\Omega} \varphi^{2}} \underset{N \rightarrow \infty}{\longrightarrow} \alpha \lambda_{1}(\Omega)
$$

and thus $\lambda_{1}^{\beta, k}(\Omega)=\alpha \lambda_{1}(\Omega)$. For $u \neq 0$ and $\lambda \leq \lambda_{1}^{\beta, k}(\Omega)$, one has therefore:

$$
E_{\lambda}(u) \geq\left(\lambda_{1}^{\beta, k}(\Omega)-\lambda\right) \int_{\Omega} u^{2} \geq 0,
$$

which in turn ensures that $S_{\lambda}(\beta, k) \geq 0$. For now, in what follows, we will not use more than this large inequality. However, once Theorem 3 is established below, it will be asserted that $S_{\lambda}(\beta, k)=E_{\lambda}(u)$ for some non-trivial $u$ and (20) will then ensure that $S_{\lambda}(\beta, k)>0$ if $\lambda<\alpha \lambda_{1}(\Omega)$.

For the sake of completeness, let us briefly investigate the larger values of $\lambda$.

Proposition 2 For $\lambda \geq \alpha \lambda_{1}(\Omega)$ and $k>0$, one has

$$
S_{\lambda}(\beta, k) \geq-\left(\lambda-\alpha \lambda_{1}(\Omega)\right) \cdot|\Omega|^{1-2 / q} .
$$

Proof. By definition of $\lambda_{1}(\Omega)$, one has:

$$
E_{\lambda}(u) \geq \int_{\Omega}|x|^{\beta}|u|^{k}|\nabla u|^{2}-\left(\lambda-\alpha \lambda_{1}(\Omega)\right) \int_{\Omega}|u|^{2} .
$$

If $\|u\|_{q}=1$ then, according to the Hölder inequality, one has $\|u\|_{2}^{2} \leq|\Omega|^{1-2 / q}$ and (21) follows immediately because $\lambda-\alpha \lambda_{1}(\Omega) \geq 0$.

Remark If $\lambda>\alpha \lambda_{1}(\Omega)+\frac{\int_{\Omega}|x|^{\beta}|\varphi|^{k}|\nabla \varphi|^{2}}{\|\varphi\|_{q}^{k}\|\varphi\|_{2}^{2}}$ where $\varphi$ is a non-trivial eigenfunction of $-\Delta \varphi=\lambda_{1}(\Omega) \varphi$ then, using $u=\varphi /\|\varphi\|_{q}$ as a test function, one gets $E_{\lambda}(u)<0$ and thus $S_{\lambda}(\beta, k)<0$. 


\subsection{Statement of the main result}

In this paper, our main result is the following.

Theorem 3 Let us assume that $n \geq 4$. One sets $q=\frac{2 n}{n-2}>2$ and assumes that

$$
0<\lambda \leq \alpha \lambda_{1}(\Omega), \quad 0 \leq k \leq q-2 \quad \text { and } \quad \beta>\frac{k n}{q}+2 .
$$

Then there exists $u \in H_{0}^{1}(\Omega)$ with $\|u\|_{L^{q}}=1$ such that $E_{\lambda}(u)=S_{\lambda}(\beta, k)$.

In section $\S 4$, one will prove a similar result about the general model (8), which will be stated as Theorem 9.

Over the course of the proof, one also reaps the following convergence result.

Proposition 4 Under the same assumptions, for any minimizing sequence $u_{j} \in H_{0}^{1}(\Omega)$ i.e. such that

$$
\left\|u_{j}\right\|_{L^{q}}=1 \quad \text { and } \quad E_{\lambda}\left(u_{j}\right)=S_{\lambda}(\beta, k)+o(1)
$$

that converges weakly to some $u \in H_{0}^{1}(\Omega)$, then $\|u\|_{L^{q}}=1$ and the convergence also holds in the strong topology of $H^{1}(\Omega)$. Moreover, $u$ is a minimizer and solves the Euler-Lagrange equation:

$$
\left\{\begin{array}{l}
-\operatorname{div}\left(\left(\alpha+|x|^{\beta}|u|^{k}\right) \nabla u\right)+\frac{k}{2}|x|^{\beta}|u|^{k-2} u|\nabla u|^{2}=\lambda u+\Theta|u|^{q-2} u \\
u_{\mid \partial \Omega}=0
\end{array}\right.
$$

for some $\Theta>0$.

Let us point out that the uniqueness of the limit is not known and constitutes a wide-open problem. For example, one knows at least that uniqueness does not hold in some cases involving weights that concentrate on multiple origins [22].

Let us also note that if $u_{j}$ is a minimizing sequence, then it is standard to check that $\left|u_{j}\right|$ is also a minimizing sequence. Thanks to Proposition 4 its limit $|u|$ is a positive nontrivial solution of (23) with an $L^{q}$-norm equal to 1 .

Let us now comment briefly upon the assumptions of the main Theorem.

- The restriction $0<\lambda \leq \alpha \lambda_{1}(\Omega)$ is natural in regard to Proposition 1 and ensures $S_{\lambda}(\beta, k) \geq 0$. For partial results in the case $\lambda>\alpha \lambda_{1}(\Omega)$, see the concluding remark of sections $\S 1.2 .2$ and $\S 3.4$.

- The restriction $\beta>\frac{k n}{q}+2$ comes from the competition between the different scalings of the terms that appear in the expression of $E_{\lambda}(u)$. In particular, the +2 offset reflects the scaling of $\int_{\Omega} u^{2}$, as pointed out in the discussion of the previous section, $\S 1.2 .1$ about (14). It is a crucial assumption that provides $S_{\lambda}(\beta, k)<\alpha S$, which will be shown in Lemma 5 below.

- The restriction $k \leq q-2$ has also been discussed in section $\S 1.2 .1$. It is necessary to ensure that the non-linear term stays of lower order throughout the minimization process, especially at the finest scales around the singularity $x=0$. Let us also observe that for the critical exponent $k=q-2$, both terms $-\operatorname{div}\left(|x|^{\beta}|u|^{k} \nabla u\right)$ and $|u|^{q-2} u$ of the Euler-Lagrange equation have the same weight for the amplitude scaling transform $u \mapsto A u$ with $A>0$.

- The case $\lambda=0$ has been extensively studied in [21] and the behavior for $\beta>\frac{k n}{q}$ is then quite different from that in Theorem 3 because the non-existence of minimizers was established. 
- It is also natural to exclude the case of $\lambda<0$. Indeed, let us consider the classical Pohožaev identity (15) when $\Omega$ is star-shaped. For $\beta \geq \frac{k n}{q}$, the left-hand side of (15) is positive but the right-hand side is negative if $\lambda<0$. There are therefore no minimizers when $\lambda<0$, at least for star-shape domains.

- When $k=0$, the non-linear nature of the problem changes. The critical value is then $\beta=2$ and is excluded. In that case, Theorem 3 contains the results obtained in [23].

Our proof of Theorem 3 follows the general principles of the method of concentration [7]. The core of our argument is a standard calculus of variation around the weak limit $u$ of a minimizing sequence, either in the direction of $u$ itself or in the direction of $\omega_{\varepsilon}$.

Thirty years later, this method can be seen as the "pedestrian way" that goes along the "highway" of the more general method of concentration-compactness using a profile decomposition [31], [27]. Let us give a rough sketch of this last method and point out the specificity of our problem that led us to choosing this rather "historical" approach.

Given a minimizing sequence $u_{k} \in H_{0}^{1}(\Omega) \cap\left\{v \mid\|v\|_{L^{q}}=1\right\}$ of some functional $F$ (in our case $F(u)=$ $\left.\int a(x, u)|\nabla u|^{2}-\lambda \int u^{2}\right)$, the boundness of the sequence in $H_{0}^{1}(\Omega)$ allows us to assume that, up to some sub-sequence, the sequence $u_{k}$ converges weakly to some limit function $u \in H_{0}^{1}(\Omega) \cap\left\{v \mid\|v\|_{L^{q}} \leq 1\right\}$. The question of showing that $u$ is a minimizer is roughly equivalent to showing that the convergence of this sequence holds in the strong topology of $L^{q}(\Omega)$ and this last statement is, at least, clearly sufficient. In case $u_{k} \nrightarrow \nrightarrow u$ in $L^{q}(\Omega)$, the concentration-compactness principle [29], [19], [24], [2], [30] provides orthogonal profiles

$$
u_{k}=u+\sum R_{j}\left(w_{j}\right)+\zeta_{k} \quad \text { with } \quad \zeta_{k} \stackrel{L^{q}}{\rightarrow} 0,
$$

where $R_{j}$ are translation-scaling operators at either different scales and/or locations and $w_{j}$ are calibrated profiles. Applying an appropriate generalized Brezis-Lieb lemma [8], [3], [30], [17] would then provide

$$
F\left(u_{k}\right)=F(u)+\sum F\left(R_{j}\left(w_{j}\right)\right)+o(1) .
$$

In most semilinear problems, the functional $F$ is invariant by translation and scaling, so the action of $R_{j}$ would commute easily with the one of $F$ and one would then get

$$
F\left(R_{j}\left(w_{j}\right)\right) \geq S_{0}
$$

where $S_{0}$ is the similar minimization problem on the whole space $\mathbb{R}^{n}$. From the a-priori comparison $0 \leq \inf F<S_{0}$ one could then deduce that the profiles just don't exist i.e. that $u_{k} \rightarrow u$ strongly in $L^{q}$. This comparison between $\inf F$ and the corresponding whole space problem is specific to each functional at hand and will be done here in Lemma 5 .

The specificity of our problem is that the weight $a(x, u)$ depends on the space variable in a nontrivial way. The whole point of our paper is to show a specific behaviour around a global minimum of $a$, under some structure assumptions. We are not convinced that one of the aforementioned generalized Brezis-Lieb lemma would indeed simplify the problem to the point of triviality.

On the contrary, a precise computation of the concentration of $u_{k}$ around the minimum of the weight $a(x, u)$ in the spirit of [7] might, at first sight, appear outdated. But, in our present case, it is more enlightening and it will thus be our course of action.

\subsection{Motivations and related questions}

The study of the general problem (7) is related to the associated Euler-Lagrange PDE which reads formally:

$$
\left\{\begin{array}{l}
-\operatorname{div}(a(x, u) \nabla u)+\frac{1}{2} \partial_{s} a(x, u)|\nabla u|^{2}=\lambda u+\Theta|u|^{q-2} u \\
u_{\mid \partial \Omega}=0
\end{array}\right.
$$


As the weight $a(x, s)$ depends in a non-trivial way in $x$ and $u$, this equation is of quasi-linear type. It only boils down to a semi-linear problem when $\partial_{s} a=0$ (i.e. when $k=0$ in the reduced model (13)), which is only one very particular case among the general assumptions of Theorem 3.

The most recents developments on quasi-linear elliptic equations seem to be focused on $p$-laplace operators i.e. a leading operator of the form $-\operatorname{div}\left(|\nabla u|^{p-2} \nabla u\right)$. Recent papers that include a critical non-linearity (see [16], [15], [18], [13]) seem to either focus on singular weights that can be controlled by a Hardy-type inequality [9]:

$$
\int_{\Omega} \frac{|u(x)|^{q^{*}}}{|x|^{s}} d x \leq C_{s}\|u\|_{H_{0}^{1}(\Omega)}^{q^{*}}
$$

or focus on a term $|u|^{p^{*}-2} u$ where $p^{*}=p n /(n-p)$ is the critical exponent associated to the $p$-Laplacian. In the case of [13], the framework is that of the Heisenberg group.

The quasilinear operator $-\operatorname{div}(a(x, u) \nabla u)$ has been studied in various papers, however not in the critical case. The paper [1] deals with a general quasilinear elliptic equation of the form

$$
-\operatorname{div}(a(x, u) \nabla u)+g(x, u, \nabla u)=\lambda h(x) u+f
$$

with a quadratic growth of $g(x, u, \nabla u)$ with respect to $\nabla u$, and very general weights $a, g$ and $h$. However, this equation does not contain a critical non-linearity like (24). In [5], the form of the quasi-linear operator gets closer to ours, but corresponds only to the case $\beta=0$ :

$$
-\operatorname{div}\left(\left(a(x)+|u|^{q}\right) \nabla u\right)+b(x) u|u|^{p-1}|\nabla u|^{2}=f \in L^{m} .
$$

This problem is truly non-linear because $p>2$ but it remains sub-critical because $m<\frac{2 n}{n+2}$. In [6], the critical points of the functional

$$
I(u)=\frac{1}{2} \int_{\Omega} a(x, u)|\nabla u|^{2}-\frac{1}{p} \int_{\Omega} u_{+}^{p}
$$

are studied for $p<2 n /(n-2)$ and a general function $a$. Again, it is a sub-critical non-linearity.

The study of the minimization problem (7) in general and of the particular form of the energy functionnal (13) is motivated by deeper questions in geometry. In [14], the minimization problem in the case $\lambda=0$ is studied on compact manifolds, along with various variants.

More generally, the Dirichlet energy associated to a map $u:(M, g) \rightarrow(N, h)$ between Riemanian manifolds takes the form

$$
\mathcal{E}(u)=\int_{M} g^{i j}(x) h_{k l}(u) \partial_{i} u^{k} \partial_{j} u^{l}(\operatorname{det}|g|)^{n / 2} d x^{1} \ldots d x^{n} .
$$

Our scalar problem with $\mathcal{E}(u)=\int_{\mathbb{R}^{n}} a(x,|u|)|\nabla u|^{2}$ and $a(x, s)=\alpha+|x|^{\beta} s^{k}$ can be seen as an entry-level model for the more general case, but with a singular metric $h$ and a non-linear term

$$
\int_{\Omega}|x|^{\beta}|u|^{k}|\nabla u|^{2}=\left(\frac{k}{2}+1\right)^{-2} \int_{\Omega}|x|^{\beta}\left(\nabla\left(|u|^{\frac{k}{2}+1}\right)\right)^{2}
$$

We refer to [25] for a general survey of those questions.

Other geometric motivations, in particular in relation to the Yamabe problem, can be found for example in [4], [10] [26], [27]. Note that the shape of the domain can have a strong influence on the type of results one can expect. See for example the seminal work of J.M. Coron [11], or [20].

Let us finally point out that the dimension $n=3$ could also be interesting for this problem, but is not yet fully understood. See [7], [12]. 


\subsection{Structure of the paper}

The paper is structured as follows. The next section, $\S 2$, proves the a-priori estimate $S_{\lambda}(\beta, k)<\alpha S$. Theorem 3, which is the main result of this paper, is proved by a contradiction argument that spans the whole of section $\S 3$. In section $\S 3.1$ one shows that the weak limit $u$ of a minimizing sequence is not zero. In sections $\S 3.2$ and $\S 3.3$, we carry out a calculus of variation around $u$, respectively in the direction of $\omega_{\varepsilon}$ defined by (2) and then along $u$ itself. The sections $\S 3.4$ and $\S 3.5$ then put the proof together.

In the final section $\S 4$, one explains how our proof could be adapted to deal with a more general minimization problem, which is stated in Theorem 9.

\section{A priori estimate on $S_{\lambda}(\beta, k)$}

Taking $\left\|\omega_{\varepsilon}\right\|_{L^{q}}^{-1} \omega_{\varepsilon}$ as a test function in $E_{\lambda}(u)$ provides a natural upper bound for $S_{\lambda}(\beta, k)$ :

$$
S_{\lambda}(\beta, k) \leq E_{\lambda}\left(\left\|\omega_{\varepsilon}\right\|_{L^{q}}^{-1} \omega_{\varepsilon}\right)
$$

We will show the following:

Lemma 5 For $n \geq 4, \lambda>0, k \geq 0$ and $\beta>\frac{k n}{q}+2$, one has:

$$
S_{\lambda}(\beta, k)<\alpha S
$$

where $\alpha$ is the minimum value of the weight in the energy functional (13).

This lemma is responsible for the main limitation on $\beta$ in Theorem 3 .

Proof. One needs to compute precisely the asymptotic expansion with respect to $\varepsilon$ of:

$$
E_{\lambda}\left(\frac{\omega_{\varepsilon}}{\left\|\omega_{\varepsilon}\right\|_{q}}\right)=E_{0}\left(\frac{\omega_{\varepsilon}}{\left\|\omega_{\varepsilon}\right\|_{q}}\right)-\frac{\lambda}{\left\|\omega_{\varepsilon}\right\|_{q}^{2}} \int_{\Omega} \omega_{\varepsilon}^{2}
$$

Each term in the expression $E_{0}\left(\frac{\omega_{\varepsilon}}{\left\|\omega_{\varepsilon}\right\|_{q}}\right)$ has been studied in our previous paper, [21]:

$$
E_{0}\left(\frac{\omega_{\varepsilon}}{\left\|\omega_{\varepsilon}\right\|_{q}}\right)=\frac{\alpha}{\left\|\omega_{\varepsilon}\right\|_{q}^{2}} \int_{\Omega}\left|\nabla \omega_{\varepsilon}\right|^{2}+\frac{1}{\left\|\omega_{\varepsilon}\right\|_{q}^{k+2}} \int_{\Omega}|x|^{\beta}\left|\omega_{\varepsilon}\right|^{k}\left|\nabla \omega_{\varepsilon}\right|^{2}
$$

with, according to (5):

$$
\frac{1}{\left\|\omega_{\varepsilon}\right\|_{q}^{2}} \int_{\Omega}\left|\nabla \omega_{\varepsilon}\right|^{2}=\frac{K_{1}+O\left(\varepsilon^{\frac{n-2}{2}}\right)}{K_{2}+o\left(\varepsilon^{\frac{n-2}{2}}\right)}=S+O\left(\varepsilon^{\frac{n-2}{2}}\right)
$$

and (see [21, Proposition 5]):

$$
\frac{1}{\left\|\omega_{\varepsilon}\right\|_{q}^{k+2}} \int_{\Omega}|x|^{\beta}\left|\omega_{\varepsilon}\right|^{k}\left|\nabla \omega_{\varepsilon}\right|^{2}= \begin{cases}O\left(\varepsilon^{\left.\frac{2 \beta-k(n-2)}{4}\right)}\right. & \text { if } \frac{k n}{q}<\beta<(k+1)(n-2) \\ O\left(\varepsilon^{\frac{(k+2)(n-2)}{4}}|\log \varepsilon|\right) & \text { if } \beta=(k+1)(n-2) \\ O\left(\varepsilon^{\frac{(k+2)(n-2)}{4}}\right) & \text { if } \beta>(k+1)(n-2) .\end{cases}
$$

Note that $(k+1)(n-2)=\frac{k n}{q}+\frac{(k+2) n}{q}$. Thanks to (6), the additional term satisfies:

$$
-\frac{\lambda}{\left\|\omega_{\varepsilon}\right\|_{q}^{2}} \int_{\Omega} \omega_{\varepsilon}^{2}=-\frac{\lambda K_{3}}{K_{2}} \begin{cases}\varepsilon|\log \varepsilon|+O(\varepsilon) & \text { if } n=4 \\ \varepsilon+O\left(\varepsilon^{\frac{n-2}{2}}\right) & \text { if } n \geq 5\end{cases}
$$


This term is clearly the dominant remainder and dictates the sign if $k>0$ and $\beta>\frac{k n}{q}+2$. Indeed, one has for $n \geq 4$ :

$$
\min \left\{\frac{2 \beta-k(n-2)}{4} ; \frac{(k+2)(n-2)}{4}\right\}>1 \Longleftrightarrow \begin{cases}\beta>\frac{k n}{q}+2 & \text { if } \beta<\frac{k n}{q}+\frac{(k+2) n}{q} \\ k>\frac{-2(n-4)}{n-2} & \text { if } \beta \geq \frac{k n}{q}+\frac{(k+2) n}{q}\end{cases}
$$

and thus

$E_{\lambda}\left(\frac{\omega_{\varepsilon}}{\left\|\omega_{\varepsilon}\right\|_{q}}\right)=\alpha S+O\left(\varepsilon^{\frac{n-2}{2}}\right)+O\left(\varepsilon^{\min \left\{\frac{2 \beta-k(n-2)}{4} ; \frac{(k+2)(n-2)}{4}\right\}}|\log \varepsilon|\right)-\frac{\lambda K_{3}}{K_{2}} \begin{cases}\varepsilon|\log \varepsilon|+O(\varepsilon) & \text { if } n=4 \\ \varepsilon+O\left(\varepsilon^{\frac{n-2}{2}}\right) & \text { if } n \geq 5 .\end{cases}$

If $k=0$, one needs to distinguish between dimensions. If $n \geq 5$, the $-\lambda$ term is dominant because $\frac{-2(n-4)}{n-2}<0$. If $n=4$, and $\beta>2$, the equivalence (26) cannot help anymore but the previous estimates directly give:

$$
E_{\lambda}\left(\frac{\omega_{\varepsilon}}{\left\|\omega_{\varepsilon}\right\|_{q}}\right)=\alpha S+O(\varepsilon)+O(\varepsilon)-\frac{\lambda K_{3}}{K_{2}} \varepsilon|\log \varepsilon|
$$

so $-\lambda$, again, dictates the sign.

Remark If $\beta=\frac{k n}{q}+2$, the non-linear term has exactly the same weight as the $-\lambda$ term so the results holds if $\lambda$ is large enough, namely

$$
C-\lambda \frac{K_{3}}{K_{2}}<0 \quad \text { i.e } \quad \frac{C K_{2}}{K_{3}}<\lambda .
$$

Sadly, the comparison between this critical value and $\alpha \lambda_{1}(\Omega)$ is not known. Note that the previous result would hold for a nontrivial range of $\lambda$ if $\alpha$ is large enough.

\section{Existence of minimizers}

Let us take a minimizing sequence $\left(u_{j}\right)_{j \in \mathbb{N}}$ for $S_{\lambda}(\beta, k)$. It is a bounded sequence in $H_{0}^{1}(\Omega)$ and one can therefore consider $u \in H_{0}^{1}(\Omega)$ a weak limit of a suitable subsequence with

$$
t=\|u\|_{L^{q}} \in[0,1]
$$

and $u_{j} \rightarrow u$ strongly in $L^{p}(\Omega)$ for any $p \in[2, q)$. Let us assume, by contradiction, that $t<1$.

\subsection{The weak limit is not identically zero}

By definition (1) of $S$, one has

$$
\alpha S \leq \alpha \int_{\Omega}\left|\nabla u_{j}\right|^{2}=S_{\lambda}(\beta, k)+\lambda \int_{\Omega} u_{j}^{2}-\int_{\Omega}|x|^{\beta}\left|u_{j}\right|^{k}\left|\nabla u_{j}\right|^{2}+o(1) .
$$

Discarding the negative term and using $u_{j} \rightarrow u$ strongly in $L^{2}$ gives

$$
\alpha S \leq S_{\lambda}(\beta, k)+\lambda \int_{\Omega} u^{2}+o(1)
$$

and thus, according to Lemma 5:

$$
\lambda \int_{\Omega} u^{2} \geq \alpha S-S_{\lambda}(\beta, k)>0
$$

which ensures that $u$ is not identically zero, and in particular that $t \neq 0$ (with $t$ defined by (27)). 


\subsection{First calculus of variations around the weak limit}

In this section, let us explore $H_{0}^{1}(\Omega)$ around the weak limit $u$ using $u+\theta \omega_{\varepsilon}$ as a test function and with $\theta$ chosen to satisfy the $L^{q}$-norm constraint.

Lemma 6 For any $v \in H_{0}^{1}(\Omega)$ such that $\|v\|_{q} \leq 1$ and $\int_{\Omega}|x|^{\beta}|v|^{k}|\nabla v|^{2}<\infty$, one has:

$$
S_{\lambda}(\beta, k) \leq E_{\lambda}(v)+\alpha S\left\{1-\|v\|_{q}^{q}\right\}^{2 / q} .
$$

For the weak limit $u$ of the above minimizing sequence $\left(u_{j}\right)_{j \in \mathbb{N}}$, one gets an equality in (28):

$$
S_{\lambda}(\beta, k)=E_{\lambda}(u)+\alpha S\left(1-t^{q}\right)^{2 / q}
$$

with $t=\|u\|_{q}$ defined by (27).

Proof of (28). If $\|v\|_{q}=1$, then $v$ is an admissible test function and (28) holds by definition. Let us now assume that $\|v\|_{q}<1$. For each $\varepsilon>0$, the intermediary values theorem ensures the existence of $c_{\varepsilon}>0$ such that $\left\|v+c_{\varepsilon} \omega_{\varepsilon}\right\|_{q}=1$. One has therefore:

$$
S_{\lambda}(\beta, k) \leq E_{\lambda}\left(v+c_{\varepsilon} \omega_{\varepsilon}\right) .
$$

The Brezis-Lieb lemma [8] allows one to compute $c_{\varepsilon}$ :

$$
1=\|v\|_{q}^{q}+c_{\varepsilon}^{q}\left\|\omega_{\epsilon}\right\|_{q}^{q}+o(1)
$$

thus

$$
c_{\epsilon}^{2}=\frac{S\left(1-\|v\|_{q}^{q}\right)^{2 / q}}{K_{1}}+o(1)
$$

Next, one computes $E_{\lambda}\left(v+c_{\varepsilon} \omega_{\varepsilon}\right)$ :

$$
S_{\lambda}(\beta, k) \leq \int_{\Omega}\left(\alpha+|x|^{\beta}\left|v+c_{\varepsilon} \omega_{\varepsilon}\right|^{k}\right)\left|\nabla\left(v+c_{\varepsilon} \omega_{\varepsilon}\right)\right|^{2}-\lambda \int_{\Omega}\left|v+c_{\varepsilon} \omega_{\varepsilon}\right|^{2} .
$$

Thus one has

$$
S_{\lambda}(\beta, k) \leq E_{\lambda}(v)+\alpha c_{\varepsilon}^{2} \int_{\Omega}\left|\nabla \omega_{\epsilon}\right|^{2}+R_{\varepsilon}
$$

with $R_{\varepsilon}=R_{\varepsilon}^{\text {sub }}+R_{\varepsilon}^{\text {crit }}$ and

$$
\begin{aligned}
& R_{\varepsilon}^{\mathrm{sub}}=2 \alpha c_{\varepsilon} \int \nabla v \cdot \nabla \omega_{\varepsilon}-2 c_{\varepsilon} \lambda \int_{\Omega} v \omega_{\varepsilon}-\lambda c_{\epsilon}^{2} \int_{\Omega} \omega_{\epsilon}^{2} \\
& R_{\varepsilon}^{\mathrm{crit}}=\int_{\Omega}|x|^{\beta}\left|v+c_{\varepsilon} \omega_{\varepsilon}\right|^{k}\left|\nabla\left(v+c_{\varepsilon} \omega_{\varepsilon}\right)\right|^{2}-\int_{\Omega}|x|^{\beta}|v|^{k}|\nabla v|^{2}
\end{aligned}
$$

and the whole point is to show that $R_{\varepsilon}=o(1)$ as $\epsilon \rightarrow 0$.

For the first term of $R_{\varepsilon}^{\text {sub }}$, one uses simply that $\omega_{\varepsilon} \rightarrow 0$ weakly in $H_{0}^{1}(\Omega)$ and strongly in $L^{2}(\Omega)$. For the next two terms of $R_{\varepsilon}^{\text {sub }}$, one combines (6) and (30) to get $O\left(\left\|\omega_{\varepsilon}\right\|_{2}\right)+O\left(\left\|\omega_{\varepsilon}\right\|_{2}^{2}\right)$ thus indeed $R_{\varepsilon}^{\text {sub }}$ converge to zero with $\varepsilon$.

All that remains is to study:

$$
\begin{aligned}
R_{\varepsilon}^{\text {crit }}= & \int_{\Omega}|x|^{\beta}\left(\left|v+c_{\varepsilon} \omega_{\varepsilon}\right|^{k}-|v|^{k}\right)|\nabla v|^{2} \\
& \quad+c_{\varepsilon}^{2} \int_{\Omega}|x|^{\beta}\left|v+c_{\varepsilon} \omega_{\varepsilon}\right|^{k}\left|\nabla \omega_{\varepsilon}\right|^{2}+2 c_{\varepsilon} \int_{\Omega}|x|^{\beta}\left|v+c_{\varepsilon} \omega_{\varepsilon}\right|^{k} \nabla v \cdot \nabla \omega_{\varepsilon} .
\end{aligned}
$$


The key is the following identity on $\mathbb{R}^{2}$ :

$$
|| x+\left.y\right|^{k}-|x|^{k} \mid \leq \begin{cases}|y|^{k} & \text { if } 0 \leq k \leq 1, \\ |y|^{k}+C_{k}\left(|x|^{k-1}|y|+|x||y|^{k-1}\right) & \text { if } k>1\end{cases}
$$

which follows respectively from || $1+\left.t\right|^{k}-\left.1|\leq| t\right|^{k}$ if $k \in[0,1]$ and $\left|\frac{|1+t|^{k}-1-|t|^{k}}{\left(1+|t|^{k-2}\right) t}\right| \leq C_{k}$ if $k \geq 0$, applied for $t=y / x \in \mathbb{R}$. Let us compute those two limits first:

$$
\begin{gathered}
\int_{\Omega}|x|^{\beta} \omega_{\varepsilon}^{k}|\nabla v|^{2}=o(1), \\
\int_{\Omega}|x|^{\beta}\left(|v|+c_{\varepsilon} \omega_{\varepsilon}\right)^{k}\left|\nabla \omega_{\varepsilon}\right|^{2}=o(1) .
\end{gathered}
$$

Subsequently, one will also check that:

$$
\begin{gathered}
\int_{\Omega}|x|^{\beta}\left|v+c_{\varepsilon} \omega_{\varepsilon}\right|^{k} \nabla v \cdot \nabla \omega_{\varepsilon}=o(1), \\
\int_{\Omega}|x|^{\beta} \omega_{\varepsilon}^{k-1}|v||\nabla v|^{2}=o(1) \quad \text { if } k>1 \\
\int_{\Omega}|x|^{\beta} v^{k-1} \omega_{\varepsilon}|\nabla v|^{2}=o(1) \quad \text { if } k>1 .
\end{gathered}
$$

Once this verification is complete, one can ascertain that $R_{\varepsilon}^{\text {crit }}$ converges to zero with $\varepsilon$ and the proof of (28) will therefore be complete.

Proof of (32). One uses an $L^{\infty} \times L^{1}$ estimate:

$$
\int_{\Omega}|x|^{\beta} \omega_{\varepsilon}^{k}|\nabla v|^{2} \leq C\left(\int_{\Omega}|\nabla v|^{2}\right) \times\left(\sup _{r \leq \delta} \frac{r^{\beta} \varepsilon^{\frac{k(n-2)}{4}}}{\left(\varepsilon+r^{2}\right)^{\frac{k(n-2)}{2}}}\right)
$$

with $\delta=\sup _{x \in \Omega}|x|$. The right-hand side is maximal around $r \sim \sqrt{\varepsilon}$ and its maximal value is of order $\varepsilon^{\frac{\beta}{2}-\frac{k(n-2)}{4}}$, which tends to zero provided

$$
\beta>\frac{k n}{q} \text {. }
$$

Proof of (33). One uses Hölder's $L^{q / k} \times L^{q /(q-k)}$ inequality with $\left|v+c_{\varepsilon} \omega_{\varepsilon}\right|^{k} \in L^{q / k}$ :

$$
\int_{\Omega}|x|^{\beta}\left|v+c_{\varepsilon} \omega_{\varepsilon}\right|^{k}\left|\nabla \omega_{\varepsilon}\right|^{2} \leq\left(\|v\|_{L^{q}}+\left\|\omega_{\varepsilon}\right\|_{L^{q}}\right)^{k} \times\left(\int_{\Omega}|x|^{\frac{\beta q}{q-k}}\left|\nabla \omega_{\varepsilon}\right|^{\frac{2 q}{q-k}} d x\right)^{1-k / q} .
$$

The first factor is bounded. The precise computation of the gradient (4) provides the necessary decay:

$$
\left(\int_{\Omega}|x|^{\frac{\beta q}{q-k}}\left|\nabla \omega_{\varepsilon}\right|^{\frac{2 q}{q-k}} d x\right)^{1-k / q} \leq C \varepsilon^{\frac{n-2}{2}}\left(\varepsilon^{-\frac{q(n-2)}{q-k}} \int_{0}^{\varepsilon} r^{\frac{\beta q}{q-k}+n-1} d r+\int_{\varepsilon}^{\delta} \frac{r^{\frac{(\beta+2) q}{q-k}+n-1}}{\left(\varepsilon+r^{2}\right)^{\frac{q n}{q-k}}} d r\right)^{1-k / q}
$$

The first term (small scale) is due to the cut-off function but is harmless because it ultimately boils down to $\varepsilon^{\beta-\frac{k n}{q}+n\left(1-\frac{1}{q}\right)}$. The second one is dealt with using a blow-up rescaling $(r=\sqrt{\varepsilon} \rho)$ :

$$
\left(\int_{\Omega}|x|^{\frac{\beta q}{q-k}}\left|\nabla \omega_{\varepsilon}\right|^{\frac{2 q}{q-k}} d x\right)^{1-k / q} \leq C\left\{\varepsilon^{\frac{n+2}{2}+\beta-\frac{k n}{q}}+\varepsilon^{\frac{n-2}{2}+\frac{1}{2}\left(\beta-\frac{k n}{q}\right)}\left(\int_{0}^{\delta / \sqrt{\varepsilon}} \frac{\rho^{\frac{(\beta+2) q}{q-k}+n-1}}{\left(1+\rho^{2}\right)^{\frac{q n}{q-k}}} d \rho\right)^{1-k / q}\right\}
$$

There are three cases: 
- If $\frac{k n}{q}<\beta<\frac{k n}{q}+n-2$, the last integral can be extended over $\mathbb{R}_{+}$and gives a harmless constant factor.

- If $\beta=\frac{k n}{q}+n-2$, the last integral is of order $\log (\delta / \sqrt{\varepsilon})$ but as $n \geq 3$, it does not prevent the whole term from tending to zero with $\varepsilon$.

- If $\beta>\frac{k n}{q}+n-2$, the last integral boils down to

$$
\left(\int_{0}^{\delta / \sqrt{\varepsilon}} \frac{\rho^{\frac{(\beta+2) q}{q-k}+n-1}}{\left(1+\rho^{2}\right)^{\frac{q n}{q-k}}} d \rho\right)^{1-k / q} \leq C \varepsilon^{\beta-\frac{k n}{q}+2}=O\left(\varepsilon^{n}\right) .
$$

In all cases, assertion (33) holds true.

Proof of (34), assuming $0 \leq k \leq 1$. Thanks to (31), it is sufficient to control the following integrals:

$$
\int_{\Omega}|x|^{\beta}|v|^{k}|\nabla v|\left|\nabla \omega_{\varepsilon}\right| \leq\left(\int_{\Omega}|x|^{\beta}|v|^{k}|\nabla v|^{2}\right)^{1 / 2} \times\left(\int_{\Omega}|x|^{\beta}|v|^{k}\left|\nabla \omega_{\varepsilon}\right|^{2}\right)^{1 / 2}
$$

whose factors are respectively bounded by assumption and controlled by (33), and

$$
\int_{\Omega}|x|^{\beta} \omega_{\varepsilon}^{k} \nabla v \cdot \nabla \omega_{\varepsilon} \leq\left(\int_{\Omega}|x|^{\beta} \omega_{\varepsilon}^{k}|\nabla v|^{2}\right)^{1 / 2} \times\left(\int_{\Omega}|x|^{\beta} \omega_{\varepsilon}^{k}\left|\nabla \omega_{\varepsilon}\right|^{2}\right)^{1 / 2}
$$

whose factors are respectively controlled by (32) and (33). This proves (34) when $k \in[0,1]$.

Proof of (35), assuming $k>1$. Let us set $\vartheta=1 / k \in] 0,1\left[\right.$. One uses Hölder's $L^{k} \times L^{k /(k-1)}$ inequality with $\left(|x|^{\beta}|\nabla v|^{2}\right)^{\vartheta}|v| \in L^{k}$ and $\left(|x|^{\beta}|\nabla v|^{2}\right)^{1-\vartheta} \omega_{\varepsilon}^{k-1} \in L^{k / k-1}$ :

$$
\int_{\Omega}|x|^{\beta} \omega_{\varepsilon}^{k-1}|v||\nabla v|^{2} \leq\left(\int_{\Omega}|x|^{\beta}|v|^{k}|\nabla v|^{2}\right)^{1 / k} \times\left(\int_{\Omega}|x|^{\beta} \omega_{\varepsilon}^{k}|\nabla v|^{2}\right)^{1-1 / k} .
$$

The first integral is bounded by assumption and the second one is controlled by (32).

Proof of (36), assuming $k>1$. Again, one uses Hölder's $L^{k} \times L^{k /(k-1)}$ inequality but this time with $\left(|x|^{\beta}|\nabla v|^{2}\right)^{\vartheta} \omega_{\varepsilon} \in L^{k}$ and $\left(|x|^{\beta}|\nabla v|^{2}\right)^{1-\vartheta} v^{k-1} \in L^{k / k-1}$ :

$$
\int_{\Omega}|x|^{\beta} v^{k-1} \omega_{\varepsilon}|\nabla v|^{2} \leq\left(\int_{\Omega}|x|^{\beta} \omega_{\varepsilon}^{k}|\nabla v|^{2}\right)^{1 / k} \times\left(\int_{\Omega}|x|^{\beta} v^{k}|\nabla v|^{2}\right)^{1-1 / k} .
$$

The first integral is controlled by (32) and the second one is bounded by assumption.

Proof of (34), assuming $k>1$. Again, one uses (31) to split the integral:

$$
\begin{aligned}
\int_{\Omega}|x|^{\beta}\left|v+c_{\varepsilon} \omega_{\varepsilon}\right|^{k}|\nabla v|\left|\nabla \omega_{\varepsilon}\right| \leq \int_{\Omega}|x|^{\beta}|v|^{k}|\nabla v|\left|\nabla \omega_{\varepsilon}\right|+c_{\varepsilon}^{k} \int_{\Omega}|x|^{\beta} \omega_{\varepsilon}^{k}|\nabla v|\left|\nabla \omega_{\varepsilon}\right| \\
+C_{k, \varepsilon}\left\{\int_{\Omega}|x|^{\beta}|v|^{k-1} \omega_{\varepsilon}|\nabla v|\left|\nabla \omega_{\varepsilon}\right|+\int_{\Omega}|x|^{\beta} v \omega_{\varepsilon}^{k-1}|\nabla v|\left|\nabla \omega_{\varepsilon}\right|\right\} .
\end{aligned}
$$

Each integral can now be controlled using Cauchy-Schwarz and the previous inequalities, namely:

$$
\begin{aligned}
\int_{\Omega}|x|^{\beta}\left|v+c_{\varepsilon} \omega_{\varepsilon}\right|^{k}|\nabla v|\left|\nabla \omega_{\varepsilon}\right| \leq & \left(\int_{\Omega}|x|^{\beta} v^{k}|\nabla v|^{2}\right)^{1 / 2} \times(33)^{1 / 2}+(32)^{1 / 2} \times(33)^{1 / 2} \\
& +C_{k, \varepsilon}\left\{(36)^{1 / 2} \times(33)^{1 / 2}+(35)^{1 / 2} \times(33)^{1 / 2}\right\} .
\end{aligned}
$$


Proof of (29). Let us now prove the second statement of Lemma 6 . One denotes by $u \in H_{0}^{1}$ a weak limit of a minimizing sequence $\left(u_{j}\right)_{j \in \mathbb{N}}$ for $S_{\lambda}(\beta, k)$. According to Fatou's lemma, one has $\int_{\Omega}|x|^{\beta}|u|^{k}|\nabla u|^{2}<\infty$ so one can apply the first part of our Lemma 6 , and thus one only needs to prove the upper bound on $E_{\lambda}(u)$. As $u_{j}$ is a minimizing sequence and $u_{j} \rightarrow u$ in $L^{2}$ :

$$
S_{\lambda}(\beta, k)+\lambda \int_{\Omega} u^{2}=\int_{\Omega}\left(\alpha+|x|^{\beta}\left|u_{j}\right|^{k}\right)\left|\nabla u_{j}\right|^{2}+o(1) .
$$

On the other hand, the classical Brezis-Lieb Lemma $\|u\|_{q}^{q}+\left\|u_{j}-u\right\|_{q}^{q}=1+o(1)$ can be rewritten

$$
\left\|u_{j}-u\right\|_{q}^{2}=\left(1-t^{q}\right)^{2 / q}+o(1)
$$

and therefore, by definition (1) of $S$ :

$$
\int_{\Omega}\left|\nabla\left(u_{j}-u\right)\right|^{2} \geq S\left\|u_{j}-u\right\|_{q}^{2}=S\left(1-t^{q}\right)^{2 / q}+o(1) .
$$

As $u_{j} \rightarrow u$ weakly in $H^{1}(\Omega)$, the left-hand side expands to

$$
\int_{\Omega}\left|\nabla\left(u_{j}-u\right)\right|^{2}=\int_{\Omega}\left|\nabla u_{j}\right|^{2}-\int_{\Omega}|\nabla u|^{2}+o(1)
$$

and thus one gets

$$
\int_{\Omega}\left|\nabla u_{j}\right|^{2} \geq S\left(1-t^{q}\right)^{2 / q}+\int_{\Omega}|\nabla u|^{2}+o(1) .
$$

Combining (37) and (38) gives:

$$
S_{\lambda}(\beta, k)+\lambda \int_{\Omega} u^{2} \geq \int_{\Omega}|x|^{\beta}\left|u_{j}\right|^{k}\left|\nabla u_{j}\right|^{2}+\alpha S\left(1-t^{q}\right)^{2 / q}+\alpha \int_{\Omega}|\nabla u|^{2}+o(1) .
$$

Fatou's lemma provides a lower bound:

$$
\int_{\Omega}|x|^{\beta}|u|^{k}|\nabla u|^{2} \leq \liminf \int_{\Omega}|x|^{\beta}\left|u_{j}\right|^{k}\left|\nabla u_{j}\right|^{2}
$$

so one gets:

$$
S_{\lambda}(\beta, k)+\lambda \int_{\Omega} u^{2} \geq \int_{\Omega}|x|^{\beta}|u|^{k}|\nabla u|^{2}+\alpha S\left(1-t^{q}\right)^{2 / q}+\alpha \int_{\Omega}|\nabla u|^{2}
$$

i.e. $S_{\lambda}(\beta, k) \geq E_{\lambda}(u)+\alpha S\left(1-t^{q}\right)^{2 / q}$. This concludes the proof of Lemma 6 .

\subsection{Second calculus of variations around the weak limit}

In this section, let us now explore $H_{0}^{1}(\Omega)$ around the weak limit $u$ using $v=(1+\theta) u$ within the lemma proved in the previous section.

Lemma 7 If $t=\|u\|_{q}<1$, the weak limit $u$ of the minimizing sequence $\left(u_{j}\right)_{j \in \mathbb{N}}$ satisfies:

$$
E_{\lambda}(u)+\frac{k}{2} \int_{\Omega}|x|^{\beta}|u|^{k}|\nabla u|^{2}=\alpha S\left(1-t^{q}\right)^{2 / q-1} \cdot t^{q} .
$$

This lemma is the Euler-Lagrange substitute within our reductio ad absurdum from the (not yet proven to be bogus) assumption that $t<1$.

Proof. The key point is that, if $t<1$, then for $\theta$ small enough one has $(1+\theta) t<1$ and thus according to Lemma 6:

$$
S_{\lambda}(\beta, k) \leq E_{\lambda}((1+\theta) u)+\alpha S\left\{1-(1+\theta)^{q} t^{q}\right\}^{2 / q}
$$


with equality when $\theta=0$. One can thus claim that:

$$
\frac{d}{d \theta} E_{\lambda}((1+\theta) u)+\left.\alpha S\left\{1-(1+\theta)^{q} t^{q}\right\}^{2 / q}\right|_{\theta=0}=0
$$

and a straightforward computation of the Taylor expansion of this expression with respect to $\theta$ gives:

$$
E_{\lambda}(u)+\frac{k}{2} \int_{\Omega}|x|^{\beta}|u|^{k}|\nabla u|^{2}=\alpha S\left(1-t^{q}\right)^{2 / q-1} \cdot t^{q},
$$

which is exactly (39).

\subsection{Proof of Theorem 3}

We are now ready to conclude the reduction ad-absurdum in which one assumed that $t=\|u\|_{q}<1$. The previous lemmas 5, 6 and 7 ensure respectively that:

$$
\begin{gathered}
S_{\lambda}(\beta, k)<\alpha S \\
S_{\lambda}(\beta, k)-\alpha S\left(1-t^{q}\right)^{2 / q}=E_{\lambda}(u) \\
E_{\lambda}(u)+\frac{k}{2} \int_{\Omega}|x|^{\beta}|u|^{k}|\nabla u|^{2}=\alpha S\left(1-t^{q}\right)^{2 / q-1} t^{q} .
\end{gathered}
$$

By definition (18) of $\lambda_{1}(\Omega)$, one has $\int_{\Omega}|\nabla u|^{2} \geq \lambda_{1}(\Omega) \int_{\Omega} u^{2}$ and thus

$$
\int_{\Omega}|x|^{\beta}|u|^{k}|\nabla u|^{2} \leq E_{\lambda}(u)+\left(\lambda-\alpha \lambda_{1}(\Omega)\right) \int_{\Omega}|u|^{2} .
$$

Let us recall that one assumes $\lambda \leq \alpha \lambda_{1}(\Omega)$ so (43) guaranties that $E_{\lambda}(u) \geq 0$. One can also discard the last negative term in (43). Combined with (41), it leads us to $S_{\lambda}(\beta, k)>0$ as long as one assumes that $t<1$.

Let us now combine (40)-(43) in one inequality with $S_{\lambda}(\beta, k)$ on both sides. More precisely, let us start by combining (42) and (43), which gives:

$$
\left(1+\frac{k}{2}\right) E_{\lambda}(u) \geq \alpha S\left(1-t^{q}\right)^{2 / q-1} t^{q}
$$

Next, one substitutes the exact value for $E_{\lambda}(u)$ given by (41):

$$
\left(1+\frac{k}{2}\right)\left(S_{\lambda}(\beta, k)-\alpha S\left(1-t^{q}\right)^{2 / q}\right) \geq \alpha S\left(1-t^{q}\right)^{2 / q-1} t^{q} .
$$

Then one uses (40) on both sides and $0<t<1$ :

$$
\left(1+\frac{k}{2}\right)\left(S_{\lambda}(\beta, k)-S_{\lambda}(\beta, k)\left(1-t^{q}\right)^{2 / q}\right)>S_{\lambda}(\beta, k) \cdot\left(1-t^{q}\right)^{2 / q-1} t^{q} .
$$

As $S_{\lambda}(\beta, k)>0$, one can simplify by $S_{\lambda}(\beta, k)$ and get an equivalent statement:

$$
1+\frac{k}{2}>\frac{\left(1-t^{q}\right)^{2 / q-1} t^{q}}{1-\left(1-t^{q}\right)^{2 / q}}
$$

We now claim that the right-hand side is an increasing function of $t$ on $(0,1)$ which is bounded from below by $q / 2$ which is the limit at the origin. Therefore, one gets a contradiction as soon as:

$$
0 \leq k \leq q-2 .
$$


To back-up our claim, let us compute the derivative:

$$
\frac{d}{d t}\left(\frac{\left(1-t^{q}\right)^{2 / q-1} t^{q}}{1-\left(1-t^{q}\right)^{2 / q}}\right)=\frac{t^{q-1}\left(1-t^{q}\right)^{-2+\frac{2}{q}}\left\{q\left(1-\left(1-t^{q}\right)^{2 / q}\right)-2 t^{q}\right\}}{\left(1-\left(1-t^{q}\right)^{2 / q}\right)^{2}}
$$

The function $q\left(1-\left(1-t^{q}\right)^{2 / q}\right)-2 t^{q}$ itself is an increasing function of $t$ on $(0,1)$ that vanishes at $t=0$. It is therefore positive which in turn implies that $\frac{\left(1-t^{q}\right)^{2 / q-1} t^{q}}{1-\left(1-t^{q}\right)^{2 / q}}$ is increasing too. The limit

$$
\lim _{t \rightarrow 0^{+}} \frac{\left(1-t^{q}\right)^{2 / q-1} t^{q}}{1-\left(1-t^{q}\right)^{2 / q}}=\lim _{x \rightarrow 0^{+}} \frac{x}{1-(1-x)^{2 / q}}=\frac{q}{2}
$$

is therefore a lower bound for the function on $(0,1)$, which settles the claim.

The general conclusion of this reduction ad-absurdum that we started p.10, is that $t=\|u\|_{q}=1$ and therefore $u$ itself is a minimizer. This concludes the proof of Theorem 3 .

Remarks on the case $\lambda>\lambda_{1}(\Omega)$. Let us briefly discuss what results survive when $\lambda$ exceeds its critical value. One can still use (43) and the previous inequality :

$$
\forall t \in(0,1), \quad\left(1-t^{q}\right)^{2 / q-1} t^{q}>\frac{q}{2}\left(1-\left(1-t^{q}\right)^{2 / q}\right) .
$$

Assuming that $S_{\lambda}(\beta, k)>0$ the previous computation boils down to:

$$
\left(\frac{q-2}{k}-1\right) \frac{1}{|\Omega|^{1-2 / q}} \frac{S_{\lambda}(\beta, k)}{\lambda-\alpha \lambda_{1}(\Omega)}<\frac{t^{2}}{1-\left(1-t^{q}\right)^{2 / q}} .
$$

But as the right-hand side is a decreasing bijective map from $(0,1)$ to $(1,+\infty)$, one gets a restriction on $t$ of the form $t<T(\Omega, \lambda)$, as long as the left-hand side exceeds 1 . In other words, one gets the following partial result that could at least be interesting for the numerical analysis of this problem.

Proposition 8 Let us assume that $n \geq 4, q=\frac{2 n}{n-2}, 0 \leq k \leq q-2, \beta>\frac{k n}{q}+2$ and that

$$
\alpha \lambda_{1}(\Omega)<\lambda<\alpha \lambda_{1}(\Omega)+\left(\frac{q-2}{k}-1\right) \frac{S_{\lambda}(\beta, k)}{|\Omega|^{1-2 / q}} \quad \text { with } \quad S_{\lambda}(\beta, k)>0 .
$$

Then for any minimizing sequence $u_{j} \in H_{0}^{1}(\Omega)$ i.e. such that

$$
\left\|u_{j}\right\|_{L^{q}}=1 \quad \text { and } \quad E_{\lambda}\left(u_{j}\right)=S_{\lambda}(\beta, k)+o(1)
$$

that converges weakly to some $u \in H_{0}^{1}(\Omega)$, then either $\|u\|_{L^{q}}=1$ and $u$ is a minimizer, or one has the alternative $\|u\|_{L^{q}} \in(0, T(\Omega, \lambda))$ where $T(\Omega, \lambda)$ is the unique solution in $(0,1)$ of

$$
\left(\frac{q-2}{k}-1\right) \frac{1}{|\Omega|^{1-2 / q}} \frac{S_{\lambda}(\beta, k)}{\lambda-\alpha \lambda_{1}(\Omega)}=\frac{T(\Omega, \lambda)^{2}}{1-\left(1-T(\Omega, \lambda)^{q}\right)^{2 / q}}
$$

\subsection{Proof of Proposition 4}

The previous proof holds for any minimizing sequence $u_{j} \in H_{0}^{1}(\Omega)$ that converges weakly to some function $u \in H_{0}^{1}(\Omega)$. From what precedes, one can claim that $\|u\|_{q}=1$ and that $u$ is a minimizer. 


\subsubsection{Strong convergence in $H^{1}(\Omega)$}

To prove Proposition 4, one first needs to show that the convergence happens in the strong topology of $H^{1}(\Omega)$. As $u$ is a minimizer, one has:

$$
\int_{\Omega}\left(\alpha+|x|^{\beta}|u|^{k}\right)|\nabla u|^{2}=\int_{\Omega}\left(\alpha+|x|^{\beta}\left|u_{j}\right|^{k}\right)\left|\nabla u_{j}\right|^{2}+o(1)
$$

and according to Fatou's lemma

$$
\int_{\Omega}|\nabla u|^{2} \leq \lim _{j \rightarrow+\infty} \int_{\Omega}\left|\nabla u_{j}\right|^{2}
$$

If this inequality were strict, then one should also have

$$
\int_{\Omega}|x|^{\beta}|u|^{k}|\nabla u|^{2}>\lim _{j \rightarrow+\infty} \int_{\Omega}|x|^{\beta}\left|u_{j}\right|^{k}\left|\nabla u_{j}\right|^{2}
$$

in order for the sum of the left-hand sides to equal the sum of the right-hand sides. However, this last one violates Fatou's lemma. One must thus have, as $\alpha>0$ :

$$
\int_{\Omega}|\nabla u|^{2}=\lim _{j \rightarrow+\infty} \int_{\Omega}\left|\nabla u_{j}\right|^{2}
$$

and the strong convergence follows from the following classical trick in Hilbert spaces:

$$
\lim _{j \rightarrow+\infty}\left\|u_{j}-u\right\|_{H^{1}}^{2}=\|u\|_{H^{1}}^{2}+\lim _{j \rightarrow+\infty}\left\|u_{j}\right\|_{H^{1}}^{2}-2 \lim _{j \rightarrow+\infty}\left(u_{j} \mid u\right)_{H^{1}}=0 .
$$

\subsubsection{Euler-Lagrange equation}

The last point of Proposition 4 is that $u$ is a non-trivial solution of the Euler-Lagrange equation.

$$
\left\{\begin{array}{l}
-\operatorname{div}\left(\left(\alpha+|x|^{\beta}|u|^{k}\right) \nabla u\right)+\frac{k}{2}|x|^{\beta}|u|^{k-2} u|\nabla u|^{2}=\lambda u+\Theta|u|^{q-2} u \\
u_{\mid \partial \Omega}=0
\end{array}\right.
$$

for some $\Theta>0$. The non-trivial part is simply that $\|u\|_{q}=1$.

For any $\varphi \in H_{0}^{1}$ and $\theta \in \mathbb{R}$, the function $\frac{u+\theta \varphi}{\|u+\theta \varphi\|_{q}}$ is an acceptable test function so

$$
S_{\lambda}(\beta, k) \leq E_{\lambda}\left(\frac{u+\theta \varphi}{\|u+\theta \varphi\|_{q}}\right) .
$$

Formally, the right-hand side can be developed as a power series in $\theta$, which takes the form:

$$
E_{\lambda}\left(\frac{u+\theta \varphi}{\|u+\theta \varphi\|_{q}}\right)=E_{\lambda}\left(\frac{u}{\|u\|_{q}}\right)+\theta \int_{\Omega} F_{\lambda}(u) \varphi+o(\theta) .
$$

If $u$ is a minimizer constructed in Theorem 3, then $\|u\|_{q}=1$ and $E_{\lambda}\left(\frac{u}{\|u\|_{q}}\right)=S_{\lambda}(\beta, k)$. To satisfy the variational inequality for any small $\theta \in \mathbb{R}$, one must then have $F_{\lambda}(u)=0$, which is the Euler-Lagrange equation. However, as $E_{\lambda}(v)=+\infty$ for some $v \in H_{0}^{1}(\Omega)$, one must be careful and check that the left-hand side is indeed a $C^{1}$ function of $\theta$ near the origin.

Let us restrict ourselves to smooth, compactly supported test functions, i.e. $\varphi \in C_{c}^{\infty}(\Omega) ; u$ is the minimizer constructed in Theorem 3. In this case, as $H_{0}^{1}(\Omega) \subset L^{q}(\Omega) \subset L^{k}(\Omega)$ for $k \leq q$, one has:

$$
\begin{aligned}
\int_{\Omega}|x|^{\beta}|u+\theta \varphi|^{k}|\nabla u+\theta \nabla \varphi|^{2} & \leq C_{k} \int_{\Omega}|x|^{\beta}\left(|u|^{k}+C\right)\left(|\nabla u|^{2}+C\right) \\
& \leq C_{k} \int_{\Omega}|x|^{\beta}|u|^{k}|\nabla u|^{2}+C_{k}^{\prime}\|u\|_{H^{1}}^{2}+C_{k}^{\prime \prime}\|u\|_{H^{1}}^{k}+C_{k}^{\prime \prime \prime} .
\end{aligned}
$$


This ensures that $E_{\lambda}\left(\frac{u+\theta \varphi}{\|u+\theta \varphi\|_{q}}\right)<\infty$ and gives meaning to the previous formal argument. To get the equation, the computation goes as follows:

$$
\|u+\theta \varphi\|_{q}^{\sigma}=\left(\int_{\Omega}|u|^{q}+\theta \int_{\Omega}|u|^{q-2} u \varphi+o(\theta)\right)^{\sigma / q}=1+\frac{\sigma \theta}{q} \int_{\Omega}|u|^{q-2} u \varphi+o(\theta)
$$

and

$$
\begin{aligned}
E_{\lambda}\left(\frac{u+\theta \varphi}{\|u+\theta \varphi\|_{q}}\right)=\| & +\theta \varphi \|_{q}^{-2}\left\{\int_{\Omega}\left(\alpha+\frac{|x|^{\beta}|u+\theta \varphi|^{k}}{\|u+\theta \varphi\|_{q}^{k}}\right)|\nabla u+\theta \nabla \varphi|^{2}-\lambda \int_{\Omega}(u+\theta \varphi)^{2}\right\} \\
= & \left(1-\frac{2 \theta}{q} \int_{\Omega}|u|^{q-2} u \varphi\right)\left\{\int_{\Omega} \alpha|\nabla u|^{2}-\lambda u^{2}+2 \theta \cdot\left(\alpha \int_{\Omega} \nabla u \nabla \varphi-\lambda \int_{\Omega} u \varphi\right)\right. \\
& \left.+\left(1-\frac{k \theta}{q} \int_{\Omega}|u|^{q-2} u \varphi\right) \cdot\left(\int_{\Omega}|x|^{\beta}\left(|u|^{k}+k \theta|u|^{k-2} u \varphi\right)\left(|\nabla u|^{2}+2 \theta \nabla u \nabla \varphi\right)\right)\right\} \\
& +o(\theta) .
\end{aligned}
$$

The term of order one in $\theta$ is:

$$
\begin{aligned}
& 2\left(\alpha \int_{\Omega} \nabla u \nabla \varphi-\lambda \int_{\Omega} u \varphi\right) \\
& \quad+2 \int_{\Omega}|x|^{\beta}|u|^{k} \nabla u \nabla \varphi+k \int_{\Omega}|x|^{\beta}|u|^{k-2} u|\nabla u|^{2} \varphi \\
& \quad-\int_{\Omega}|u|^{q-2} u \varphi\left\{\frac{2}{q}\left(\int_{\Omega} \alpha|\nabla u|^{2}-\lambda u^{2}\right)+\left(\frac{k+2}{q}\right) \int_{\Omega}|x|^{\beta}|u|^{k}|\nabla u|^{2}\right\}=\int_{\Omega} F_{\lambda}(u) \varphi .
\end{aligned}
$$

with $\frac{1}{2} F_{\lambda}(u)=-\alpha \Delta u-\lambda u-\operatorname{div}\left(|x|^{\beta}|u|^{k} \nabla u\right)+\frac{k}{2}|x|^{\beta}|u|^{k-2} u|\nabla u|^{2}-\Theta|u|^{q-2} u$ and

$$
\Theta=\frac{2}{q}\left(\int_{\Omega} \alpha|\nabla u|^{2}-\lambda u^{2}\right)+\left(\frac{k+2}{q}\right) \int_{\Omega}|x|^{\beta}|u|^{k}|\nabla u|^{2}
$$

thus $\Theta>0$ for $\lambda \leq \alpha \lambda_{1}(\Omega)$ and $u \not \equiv 0$.

\section{Generalisations and open problems}

Theorem 3 remains valid for the following minimisation problem, which is more general.

Theorem 9 Let us consider:

$$
S_{\lambda}(a)=\inf _{\substack{u \in H_{0}^{1}(\Omega) \\\|u\|_{L^{q}=1}}}\left\{\int_{\Omega} a(x, u(x))|\nabla u(x)|^{2} d x-\lambda \int_{\Omega} u^{2}\right\}
$$

where $a(x, s)=b_{1}(x)+b_{2}(x)|s|^{k}$. The functions $b_{1}, b_{2}$ satisfy the following assumptions:

1. $b_{1}$ has a global minimum $\alpha=b_{1}\left(x_{0}\right)$ at some point $x_{0} \in \Omega$, of order $\gamma>2$, i.e.:

$$
\left\{\begin{array}{l}
b_{1}(x)=\alpha+O\left(\left|x-x_{0}\right|^{\gamma}\right), \quad \gamma>2 \\
b_{1}(x) \geq \alpha \quad \text { if } \quad x \neq x_{0}
\end{array}\right.
$$

2. $b_{2}$ is positive and has a unique zero in $\Omega$ at the same point $x_{0}$, of order $\beta$

$$
\left\{\begin{array}{l}
b_{2}(x)=\left|x-x_{0}\right|^{\beta}+o\left(\left|x-x_{0}\right|^{\beta}\right) \\
b_{2}(x)>0 \quad \text { if } \quad x \neq x_{0}
\end{array}\right.
$$


One assumes restrictions on the parameters that are similar to the ones in Theorem 3:

$$
0<\lambda \leq \alpha \lambda_{1}(\Omega), \quad 0 \leq k \leq q-2 \quad \text { and } \quad \beta>\frac{k n}{q}+2 .
$$

Then there exists $u \in H_{0}^{1}(\Omega)$ with $\|u\|_{L^{q}}=1$ such that

$$
S_{\lambda}(a)=\int_{\Omega} a(x, u(x))|\nabla u(x)|^{2} d x-\lambda \int_{\Omega} u^{2}
$$

Remark. Actually, the result (and our proof) would remain valid for a general function $a(x, s)$ such that:

$$
b_{1}^{-}(x)+b_{2}^{-}(x)|s|^{k} \leq a(x, s) \leq b_{1}^{+}(x)+b_{2}^{+}(x)|s|^{k}
$$

with $b_{1}^{ \pm}$and $b_{2}^{ \pm}$that satisfy similar assumptions.

Sketch of proof. Let us focus briefly on how one would adapt the previous proof to deal with this case. The first crucial step is the comparison between $S_{\lambda}(a)$ and $\alpha S$ (Lemma 5). The remainder of $b_{1}$ produces an additional term

$$
\int_{\Omega}\left|x-x_{0}\right|^{\gamma}\left|\nabla\left(\frac{\omega_{\varepsilon}}{\left\|\omega_{\varepsilon}\right\|_{q}}\right)\right|^{2}=O\left(\varepsilon^{\gamma / 2}\right)
$$

which is negligible in comparison to $-\lambda \int_{\Omega}\left(\frac{\omega_{\varepsilon}}{\left\|\omega_{\varepsilon}\right\|_{q}}\right)^{2} \sim-C \lambda \varepsilon$ provided $\gamma>2$. The second crucial step is to prove (29). Provided $b_{1}(x) \geq b_{1}\left(x_{0}\right)=\alpha$, i.e. that the minimum at $x_{0}$ is a global one, one still gets :

$$
\int_{\Omega} b_{1}(x)\left|\nabla u_{j}\right|^{2} \geq \alpha S\left(1-t^{q}\right)^{2 / q}+\int_{\Omega} b_{1}(x)|\nabla u|^{2}+o(1)
$$

instead of (38) and the rest of the proof remains unchanged.

Further generalisations beyond Theorem 9, or at best (54), seem for now out of reach.

- If $b_{1}$ admits a minimum of order $\gamma \leq 2$ at $x_{0}$, the comparison between $S_{\lambda}(a)$ and $\alpha S$ is not clear anymore.

- If $b_{1}$ admits only a local minimum at $x_{0}$ which is not global, most of the comparisons that we used cease to be true.

- If the zero of $b_{2}$ occurs at a point $x_{1}$ distinct of the point $x_{0}$ where $b_{1}$ reaches its minimum, then minimizing sequences can either concentrate around $x_{0}$ and one expect a behaviour similar to the model case with $\beta=0$ (i.e. the solution is in the linear regime studied in [21] and a minimizer should exist), or the the minimizing sequence can concentrate around $x_{1}$ and in that case again, it is not clear how to compare $S_{\lambda}(a)$ and $(\min a) \cdot S$ anymore.

\section{References}

[1] Abdellaoui, B.; Boccardo, L.; Peral, I.; Primo, A. Quasilinear elliptic equations with natural growth. Differential Integral Equations 20 (2007), no. 9, 1005-1020.

[2] Adimurthi; Tintarev, C., On compactness in the Trudinger-Moser inequality. Ann. Sc. Norm. Super. Pisa Cl. Sci. (5) 13 (2014), no. 2, 399-416.

[3] Adimurthi ; Tintarev, C., On the Brezis-Lieb lemma without pointwise convergence. NoDEA Nonlinear Differential Equations Appl. 22 (2015), no. 5, 1515-1521. 
[4] Aubin, T., Equations différentielles non linéaires et problème de Yamabe concernant la courbure scalaire. J. Math. Pures Appl. (9) 55 (1976), no. 3, 269-296.

[5] Boccardo, L., A contribution to the theory of quasilinear elliptic equations and application to the minimization of integral functionals. Milan J. Math. 79 (2011), no. 1, 193-206.

[6] Boccardo, L.; Pellacci, B., Bounded positive critical points of some multiple integrals of the calculus of variations. Nonlinear equations: methods, models and applications (Bergamo, 2001), 33-51, Progr. Nonlinear Differential Equations Appl., 54, Birkhäuser, Basel, (2003).

[7] Brézis, H.; Nirenberg, L., Positive solutions of nonlinear elliptic equations involving critical Sobolev exponents. Comm. Pure Appl. Math. 36 (1983), no. 4, 437-477.

[8] Brézis, H.; Lieb, E., A relation between pointwise convergence of functions and convergence of functionals. Proc. Amer. Math. Soc. 88 (1983), no. 3, 486-490.

[9] Caffarelli, L.; Kohn, R. and Nirenberg, L., First order interpolation inequalities with weights, Compositio Math. 53, (1984), p. 259-275.

[10] Cordero-Erausquin, D.; Nazaret, B.; Villani, C., A mass-transportation approach to sharp Sobolev and Gagliardo-Nirenberg inequalities. Adv. Math. 182 (2004), no. 2, 307-332.

[11] Coron, J.-M., Topologie et cas limite des injections de Sobolev. C. R. Acad. Sci. Paris Sér. I Math. 299 (1984), no. 7, 209-212.

[12] Crouau, R.; Hadiji, R.; Lewandowski, R., Critical Sobolev exponent and the dimension three. Houston J. Math. 18 (1992), no. 2, 189-204.

[13] D'Ambrosio, L.; Mitidieri, E., Quasilinear elliptic equations with critical potentials. Adv. Nonlinear Anal. 6 (2017), no. $2,147-164$.

[14] Demyanov, A.V.; Nazarov A.I., On the existence of an extremal function in Sobolev embedding theorems with a limit exponent. (Russian) Algebra i Analiz 17 (2005), no. 5, 105-140; translation in St. Petersburg Math. J. 17 (2006), no. 5, 773-796

[15] de Valeriola, S.; Willem, M., On some quasilinear critical problems. Adv. Nonlinear Stud. 9 (2009), no. 4, 825-836.

[16] Drábek, P.; Huang, Y.X., Multiplicity of positive solutions for some quasilinear elliptic equation in RN with critical Sobolev exponent. J. Differential Equations 140 (1997), no. 1, 106-132.

[17] Emelyanov, E. Yu.; Marabeh, M. A. A., Two measure-free versions of the Brezis-Lieb lemma. Vladikavkaz. Mat. Zh. 18 (2016), no. 1, 21-25.

[18] Faraci, F.; Farkas, C., A quasilinear elliptic problem involving critical Sobolev exponents. Collect. Math. 66 (2015), no. 2, 243-259.

[19] Gérard, P., Description du défaut de compacité de l'injection de Sobolev. ESAIM Control Optim. Calc. Var. 3 (1998), 213-233.

[20] Hadiji, R., Solutions positives de l'équation $-\Delta u=u^{p}+\mu u^{q}$ dans un domaine à trou. Ann. Fac. Sci. Toulouse Math. (5) 11 (1990), no. 3, 55-71.

[21] Hadiji, R.; Bae,S ; Vigneron, F. ; Yazidi, H., A Nonlinear Existence Result for a Quasi-Linear Elliptic PDE. Journal of mathematical Analysis and Applications, 396 (2012), no 1, 98-107.

[22] Hadiji, R.; Molle, R.; Passaseo, D.; Yazidi, H., Localization of solutions for nonlinear elliptic problems with critical growth. C. R. Math. Acad. Sci. Paris 343 (2006), no. 11-12, 725-730.

[23] Hadiji, R.; Yazidi, H., Problem with critical Sobolev exponent and with weight. Chin. Ann. Math. Ser. B 28 (2007), no. 3, 327-352. 
[24] Jaffard, S., Analysis of the lack of compactness in the critical Sobolev embeddings. J. Funct. Anal. 161 (1999), no. 2, 384-396.

[25] Jost, J., Riemannian Geometry and Geometrical Analysis. Springer (1998).

[26] Lee, J.M.; Parker, T.H., The Yamabe problem. Bull. Amer. Math. Soc. 17 (1987), no. 1, 37-91.

[27] Lions, P. L., The concentration-compactness principle in the calculus of variations. The limit case, Part 1, Revista Mat. Iberoamericana 1, no 1, (1985), pp. 145-201 ; Part 2, Revista Mat. Iberoamericana 1, no 2, (1985), pp. 45-121.

[28] Pohožaev, S. I., On the eigenfunctions of the equation $\Delta u+\lambda f(u)=0$. Dokl. Akad. Nauk SSSR 165 (1965), $36-39$.

[29] Solimini, S., A note on compactness-type properties with respect to Lorentz norms of bounded subsets of a Sobolev space. Ann. Inst. H. Poincaré Anal. Non Linéaire 12 (1995), no. 3, 319-337

[30] Solimini, S.; Tintarev, C., Concentration analysis in Banach spaces. Commun. Contemp. Math. 18 (2016), no. 3 .

[31] Struwe, M., A global compactness result for elliptic boundary value problems involving limiting nonlinearities, Math. Z., 187, (1984), pp. 511-517. 